\title{
Myointimal hyperplasia of the mesenteric veins mimicking infectious colitis
}

\author{
Benjamin S. Thomas
}

Accepted: 15 June 2012 / Published online: 26 June 2012

(C) Springer-Verlag 2012

\section{Dear Editor:}

I read with great interest the letter written by Feo et al. where they described a case of idiopathic myointimal hyperplasia of the mesenteric veins (IMHMV) in an elderly patient. I also experienced a challenging case in an older gentleman which resulted in the rare diagnosis of IMHMV.

A 62-year-old previously healthy male was transferred from a Micronesian hospital for evaluation of recurrent bloody diarrhea. Three weeks prior, Entamoeba histolytica was identified by stool microscopy and Escherichia coli (non-O157:H7) by stool culture. He was treated with ciprofloxacin and metronidazole $(\times 10$ days $)$ with temporary resolution of his symptoms. At our institution, the patient was having guaiac-positive, small-volume stools. Physical examination revealed a large, tender palpable mass in the left lower quadrant. Initial laboratory studies were normal other than mild anemia (Hgb $12.5 \mathrm{~g} / \mathrm{dL}$ ). Repeat stool studies for pathogenic bacteria and parasites were negative. CT scan of the abdomen showed extensive wall thickening of the entire rectum and sigmoid colon. The patient underwent colonoscopy where circumferential ulcerations were visualized at $15-50 \mathrm{~cm}$ from the rectum. Intravenous metronidazole and ceftriaxone as well as oral paromomycin were administered and the patient was discharged home after 7 days. During outpatient follow up 1 week later, the patient complained of recurrent abdominal pain and bloody diarrhea. Physical examination revealed significant left lower quadrant abdominal pain with rebound tenderness. Clinical deterioration warranted re-admission to the hospital and an exploratory laparotomy with sigmoidectomy was performed. Pathologic

\footnotetext{
B. S. Thomas $(\bowtie)$

Department of Medicine, John A. Burns School of Medicine,

University of Hawaii,

1356 Lusitana Street, 7th Floor,

Honolulu, HI 96813, USA

e-mail: bsthomas@hawaii.edu
}

examination of the resected colon segment showed prominent myointimal hyperplasia of the mesenteric veins with mesenteric fibrosis and fat necrosis.

As noted by Feo et al., IMHMV is a nonthrombotic occlusion of the mesenteric veins secondary to myointimal hyperplasia. In the past, some authors have postulated that IMHMV and mesenteric inflammatory veno-occlusive disease (MIVOD) are just a spectrum of the same disorder. The etiology of both conditions is unknown and they have both been reported in association with heterogeneous array of conditions. The importance of recognizing both rare disorders is highlighted by the method of and response to treatment. Distinctive demographic characteristics, disease distribution patterns, and histologic findings in the two disorders are present (noted by Feo et al. in their letter), but interestingly, the treatment (surgical resection of the affected bowel) is the same. And additionally, the outcome of treatment is nearly identical. There is one case of recurrent MIVOD, which could have been the result of a lack of complete resection.

With so few cases reported in each disease, it is difficult to make definitive comparisons between the two disorders; although, with each additional case of IMHMV reported in older patients, the age demographic is becoming more similar. IMHMV was previously described only in males, but the recent case presented by Feo et al. was female (MIVOD has roughly equal sex distribution). Rarely suspected and probably underreported, IMHMV should be in the differential diagnosis in patients presenting with abdominal pain and bloody diarrhea and our evolving knowledge of the demographic features may assist in recognizing its presence.

Declaration of interest No funding was associated with this study. The author has no commercial relationship or potential conflict of interest related to the submission of this manuscript. 